\title{
Magnetic Resonance Imaging of DNP Enhancements in a Rotor Spinning at the Magic Angle
}

Frédéric A. Perras, ${ }^{a}$ Takeshi Kobayashi, ${ }^{\mathrm{a}}$ and Marek Pruski ${ }^{\mathrm{a}, \mathrm{b} *}$

${ }^{a}$ U.S. DOE Ames Laboratory, Ames, IA 50011-3020, USA

${ }^{\mathrm{b}}$ Department of Chemistry, Iowa State University, Ames, IA 50011-3020, USA

${ }^{*}$ Corresponding author.

M. Pruski: Ames Laboratory, Iowa State University, 230 Spedding Hall, Ames, IA 50011-3020, USA. Phone: +1 5152942017 Fax: +1 515294 4709. E-mail address: mpruski@iastate.edu.

\section{Abstract}


Simulations performed on model, static, samples have shown that the microwave power is non-uniformly distributed in the magic angle spinning (MAS) rotor when using conventional dynamic nuclear polarization (DNP) instrumentation. Here, we applied the stray-field magic angle spinning imaging (STRAFI-MAS) experiment to generate a spatial map of the DNP enhancements in a full rotor, which is spun at a low rate in a commercial DNP-MAS NMR system. Notably, we observed that the enhancement factors produced in the center of the rotor can be twice as large as those produced at the top of the rotor. Surprisingly we observed that the largest enhancement factors are observed along the axis of the rotor as opposed to against the walls of the rotor, which are most directly irradiated by the microwave beam. We lastly observed that the distribution of enhancement factors can be moderately improved by degassing the sample and increasing the microwave power. The inclusion of dielectric particles greatly amplifies the enhancement factors throughout the rotor. The STRAFI-MAS approach can provide useful guidance for optimizing the access of microwave power to the samples, and thereby lead to further increases in sensitivity of DNP-MAS NMR.

\section{Graphical Abstract}

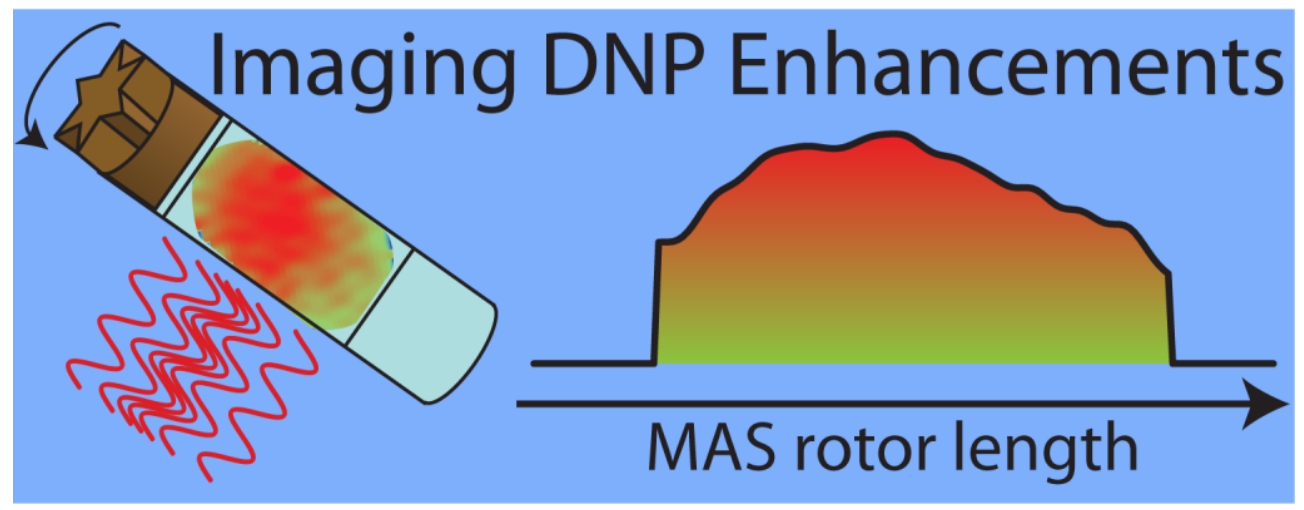




\section{Introduction}

Dynamic nuclear polarization (DNP) has gained a considerable importance in recent years as a method for enhancing nuclear magnetic resonance (NMR) lines.[1,2,3,4,5,6] Simply put, DNP involves the irradiation of unpaired electrons with microwaves at their electron paramagnetic resonance (EPR) frequency in order to transfer their much greater polarization to the nuclei. Exogenous stable free radicals are normally used as a source of unpaired electrons. The magnitude of the theoretical maximum sensitivity gain is on the order of the ratio of the electron and nuclear magnetogyric ratios $\left(\gamma_{\mathrm{e}} / \gamma_{\mathrm{H}}=658\right.$, for example). As DNP-NMR data are typically acquired at cryogenic temperatures, to slow the electrons' relaxation times, the enhancement factors can in some cases exceed 4 orders of magnitude, when compared to room temperature data.[7] These unprecedented sensitivity gains open the door to efficiently studying unreceptive nuclides,[8,9] minute sample quantities,[10] and prohibitively small surface areas.[11]

DNP has branched into principally two distinct techniques. The first, dissolution DNP,[7] involves the hyperpolarization of the sample at cryogenic temperatures followed by its rapid dissolution in a warm solvent and the acquisition of a liquid-state NMR spectrum. The second DNP technique, one of relevance to the present study, involves solid-state NMR (SSNMR) under magic-angle spinning (MAS).[2] In a DNP-MAS experiment, the sample is spun at the magic angle at a low temperature $(<110 \mathrm{~K})$, and is irradiated in situ by high power microwaves produced by a gyrotron.[12,13] This is an attractive technique because it can boost the sensitivity of SSNMR experiments using a conventional spectrometer equipped with the lowtemperature MAS and gyrotrons accessories.[14] 
DNP-MAS equipment, however, does not allow for an optimal irradiation of the sample with microwaves. Power is lost while the microwaves are transmitted through a long waveguide connecting the gyrotron and the NMR probe. The microwaves must then pass between the turns of the windings of the radiofrequency coil and penetrate the wall of the rotor before irradiating the sample itself. Simulations of the microwave propagation in the stator of a DNP-MAS probe have shown that the resulting distribution of microwaves is far from uniform throughout the sample.[15,16,17] The microwave beam diffracts inside the rotor after passing through the windings of the coil and is reflected by the stator, creating "hot spots" of larger microwave power in the rotor. Note that microwave diffraction patterns are not expected within an MAS rotor due to the minute variations in the pitch of the coil that are present.[17] Such calculations predict that the largest microwave power, and thus DNP enhancement, is observed along the walls of the rotor near the area where the microwave beam enters the sample. However, the important effects of sample spinning, which would serve to spatially average some of the anisotropies in the microwave power, are not included in the simulations.[17] It would be

preferential to instead experimentally map the microwave power, or similarly the DNP enhancement factors as the two are correlated,[18] inside the rotor under typical experimental conditions.

\section{STRAFI-MAS}

In 2005 Baltisberger and co-workers indeed presented a simple magnetic resonance imaging (MRI) experiment that could be performed on any MAS probe.[19] Instead of using pulsed magnetic field gradients to spatially encode the NMR signals, they took advantage of the large stray field gradients that are present when the probe is lowered from its optimal height. Although this gradient is continuously oriented along the ' $z$ ' direction, the sample rotation in an 
MAS probe can be used to reorient the sample such that the gradient is sequentially aligned along all three Cartesian directions.[19,20,21,22] The pulse sequence diagram for the twodimensional (2D) cross-polarization (CP) stray-field imaging (STRAFI)-MAS experiment is shown in Figure 1. It can be seen that, in the rotor frame, the direction of the gradient is modulated such that it is aligned along three orthogonal directions every $120^{\circ}$ of rotation (or $1 / 3$ of the rotor period: $\tau_{R}$ ). If the spinning frequency is slow compared to the size of the gradient and the acquisition times, the evolution of the signal can be approximated as being under the influence of a static gradient. The orientation of the rotor, and thus the direction of the gradient, is first encoded when the desired spins are polarized by means of cross-polarization. The initial direction of the gradient, following an optical trigger and the end of the spin lock, is arbitrarily chosen as being along $\mathrm{x}$. Following the evolution of the sample under a gradient centered on $\mathrm{G}_{\mathrm{x}}$, a z-filter is applied to store the magnetization while the sample is reoriented. A spin echo is then produced to detect the NMR signal with the gradient aligned along z. A 2D Fourier transform yields a $2 \mathrm{D}$ image of the contents of the rotor.

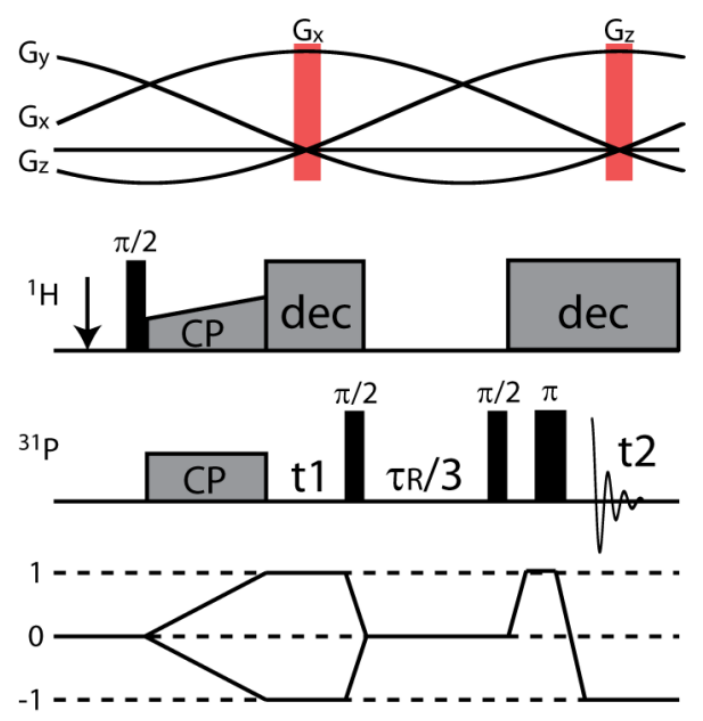


Figure 1. 2D CP-STRAFI-MAS pulse sequence diagram. The stray field gradient is aligned with the applied magnetic field and thus the gradient strengths oscillates between the three Cartesian axes of the rotor every $120^{\circ}$, as shown at the top. Red boxes highlight the evolution periods along $G_{x}$ and $G_{z}$ for the 2D CP-STRAFI-MAS experiment. Following an optical trigger to encode the rotor's orientation, magnetization is transferred from ${ }^{1} \mathrm{H}$ to ${ }^{31} \mathrm{P}$ spins using $\mathrm{CP}$. The magnetization then evolves under a $G_{x}$ gradient, and is stored along $B_{0}$ until the gradient is aligned in the $\mathrm{z}$ direction and a spin echo is acquired. Phase cycling is chosen to select the coherence transfer pathway shown at the bottom.

All that is necessary to spatially map the DNP enhancement factors in the rotor is to acquire CP-STRAFI-MAS images with the microwave irradiation turned on and off ('MW on' and 'MW off'). By dividing the DNP image with a non-enhanced image, where both were acquired sequentially under identical experimental and processing conditions, a 2D image of the enhancement factors in the rotor can be obtained. This process also counters the distortions occurring from the non-linearity of the magnetic field gradient, which emphasizes the sample located near the cap. Similarly, smearing distortions caused by the finite sample rotation are not expected to impact the enhancement images, as these are present in both the 'MW on' and 'MW off' images.

\section{Experimental Setup}

All DNP-MAS experiments were performed using a commercial Bruker MAS-DNP system equipped with a Bruker AVANCE III console, a $264 \mathrm{GHz}$ gyrotron, and a 3.2-mm low temperature MAS probe. Performing STRAFI-MAS experiments using a DNP-MAS probe 
presents several challenges. Firstly, the probe cannot be lowered indefinitely so as to maximize the field gradient and thus the spatial resolution. The necessary connection to the waveguide transmitting the microwaves limits the degree to which the probe may be lowered. Additionally, as the probe is pulled out, the magnetic field is reduced further from the optimum field for DNP. By removing one of the connections of the probe to the waveguide we were able to lower the probe by $42 \mathrm{~mm}$ and achieve a field gradient of $\sim 100 \mathrm{mT} / \mathrm{m}$. This corresponds to a spread of resonance frequencies of $\sim 40 \mathrm{ppm}$ throughout the rotor. The gradient strength was determined by measuring the ${ }^{1} \mathrm{H}$ resonance frequency of water as the probe is lowered in a controlled fashion. The remaining pieces of the waveguide were brought into contact with the use of a custom aluminum adapter and sealed with parafilm.

The second hurdle pertains to the choice of sample, which should involve a spin-1/2 nucleus with a relatively high Larmor frequency, to obtain a large frequency dispersion in the image, yet a small static linewidth. A sample containing ${ }^{1} \mathrm{H}$ is required to obtain large DNP enhancements, however, ${ }^{1} \mathrm{H}$ cannot be directly detected, due to its broad static linewidth which by far exceeds $40 \mathrm{ppm}$. Also impractical is the use of ${ }^{13} \mathrm{C},{ }^{15} \mathrm{~N}$, and ${ }^{29} \mathrm{Si}$ spins, in this case due to low value of $\gamma$ and large chemical shift anisotropy. We thus chose to exploit ${ }^{31} \mathrm{P}$ nuclei in sodium phosphate dodecahydrate $\left(\mathrm{Na}_{3} \mathrm{PO}_{4} \cdot 12 \mathrm{H}_{2} \mathrm{O}\right)$, which combine high sensitivity (especially under ${ }^{1} \mathrm{H}$ ${ }^{31} \mathrm{P} \mathrm{CP}$ ) with small chemical shift anisotropy and small homonuclear dipolar coupling. Indeed, the static ${ }^{31} \mathrm{P}$ linewidth in $\mathrm{Na}_{3} \mathrm{PO}_{4} \cdot 12 \mathrm{H}_{2} \mathrm{O}$ under ${ }^{1} \mathrm{H}$ decoupling at $100 \mathrm{~K}(1 \mathrm{kHz})$ corresponds to a spatial resolution of approximately $0.5 \mathrm{~mm}$, which is sufficient to map the DNP enhancements. Although a higher resolution would be desired, achieving the necessary slow rotation frequencies to reach this resolution while maintaining a low temperature would be highly challenging. 
To generate the images, $\mathrm{Na}_{3} \mathrm{PO}_{4} \cdot 12 \mathrm{H}_{2} \mathrm{O}$ was powdered, wetted with a $16 \mathrm{mM}$ solution of the TEKPol biradical in tetrachloroethane (TCE),[23] and packed in a 3.2-mm o.d. sapphire rotor. The rotor was then sealed with a Teflon plug (see Figure 2) and capped with a specially designed fluteless cap that enables stable spinning at low MAS frequencies,[24] as is common for STRAFI-MAS experiments.[20,21,22] The sample was then cooled to approximately $105 \mathrm{~K}$ and spun at $143 \mathrm{~Hz}$ with a stability of $\pm 1 \mathrm{~Hz}$. The STRAFI-MAS images were acquired using a $2.35 \mu \mathrm{s}{ }^{1} \mathrm{H} 90^{\circ}$ pulse, a $2 \mathrm{~ms}$ contact time, ${ }^{1} \mathrm{H}$ decoupling during $\mathrm{t}_{1}$ and $\mathrm{t}_{2}$, and 3.75 and $7.5 \mu \mathrm{s}{ }^{31} \mathrm{P}$ 90 and $180^{\circ}$ pulses. The spectra were acquired in 24 rows, with $\Delta t_{1}$ of $50 \mu$ s, using 8 scans per row with a recycle delay of 1 minute. The digital resolution was increased to 64 points by zero filling. Note that during the entire evolution time of $24 \Delta t_{1}$, the rotor rotates by approximately $60^{\circ}$. To minimize the resulting image distortions, the center of the evolution period was fixed.[19] Purely absorptive phase 2D lineshapes were obtained using the States procedure.[25]

Images acquired with 'MW on' and 'MW off' were processed identically and then divided by each other using an in-house $\mathrm{C}$ program in order to obtain an image of the enhancement factors (see Figure 2). In order to avoid dividing by pure noise, only data points where the signal is greater than $20 \%$ of the maximum intensity were kept, the remaining data points were set to zero. The images were generated using DMFit.[26] The spatial resolution is highlighted in an image acquired for a rotor containing only $1 \mathrm{~mm}$ of sample at the bottom (Figure 2d). 

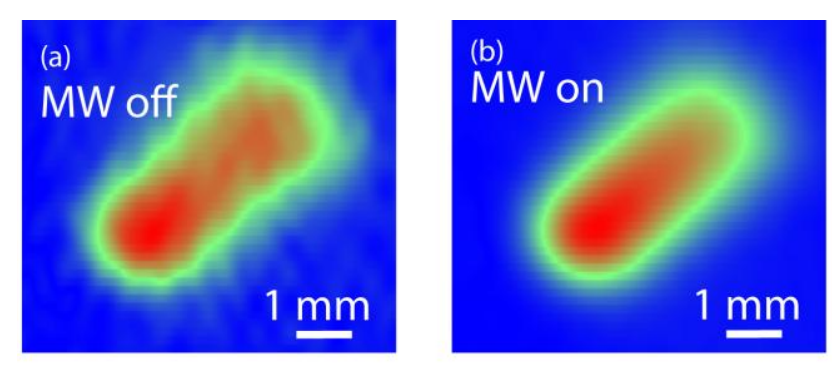

(e)
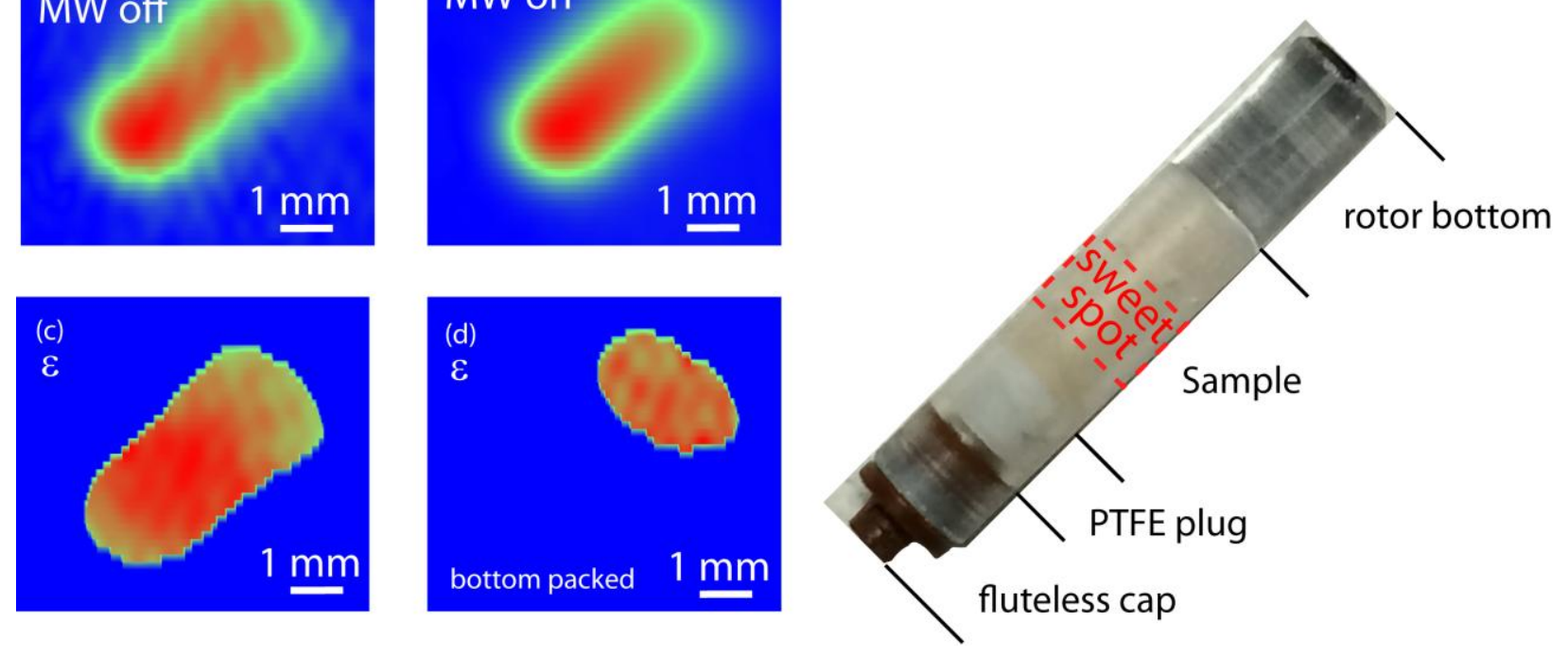

Figure 2. A pictorial description of the process for obtaining 2D DNP enhancement images. 'MW off' (a) and 'MW on' (b) CP-STRAFI-MAS images are sequentially acquired under identical conditions. The 'MW on' image is then divided by the 'MW off' image (only the signals having an intensity greater than a cut-off value of $20 \%$ are kept to non-zero) to produce a DNP enhancement image (c) free of distortions from the non-linearity of the gradient and the finite sample spinning. An enhancement image of a sample packed in the lowest $1 \mathrm{~mm}$ of the rotor is shown in (d). The images are oriented as shown in (e). Note also that a fluteless cap is used to maintain stable spinning at low frequencies.

\section{Results and Discussion}

Images have been acquired using a full rotor containing $\mathrm{Na}_{3} \mathrm{PO}_{4} \cdot 12 \mathrm{H}_{2} \mathrm{O}$ and a $16 \mathrm{mM}$ solution of TEKPol[23], as explained above. The stray field, of course, increases towards the center of the magnet and thus towards the cap. Given that the images are plotted as conventional 2D NMR spectra, the highest resonance frequency is found at the bottom left of the image and thus the images show the rotor oriented with its cap facing downwards (Figure 2e). 
By viewing the images it becomes apparent that the DNP enhancement factors are far from uniform inside the rotor. Firstly, there is a hot spot in the center of the rotor where the enhancement factors are largest. Contrarily to the simulations performed on static rotors that predict a larger enhancement factor along the walls of the rotor, $[15,16,17]$ we actually observe that the enhancement factor is approximately $25 \%$ smaller along the walls of the rotor than it is along its axis. It is likely that performing a spatial average of the microwave power, in accordance with sample spinning, would reproduce this experimental result in silico, as noted by Maly and Sirigiri.[17] We also notice, in accordance with previous simulations, $[15,16,17]$ that lengthwise the enhancement factors are largest in the center of the rotor where the microwave beam irradiates the sample. Indeed, a slice taken along the rotation axis of the rotor, (Figure 3) demonstrates that the enhancement factor at the bottom and top of the rotor $(\varepsilon \sim 3)$ can be lower by a factor of 2 compared to the center of the rotor $(\varepsilon \sim 6)$. This result sheds light on the reason why fully packing a DNP rotor leads to a lower average enhancement, when compared to only using $2 / 3$ of the rotor's active volume. Note that, although DNP enhancement factors are largest for center-packed rotors, the highest overall sensitivity would be provided by a full rotor. A Gaussian shape with a width at half height of $5.66 \mathrm{~mm}$, which corresponds to the thickness of the microwave beam produced by our gyrotron, is also plotted in Figure 3a. As can be seen, the enhancement profile closely matches the microwave power profile from the instrument's specifications. Like the enhancement factors, the microwave power is also halved at the top and bottom of the sample. 
(a) prior to degassing

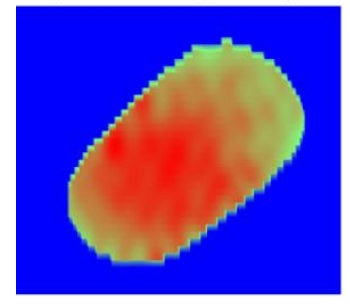

(b) after degassing
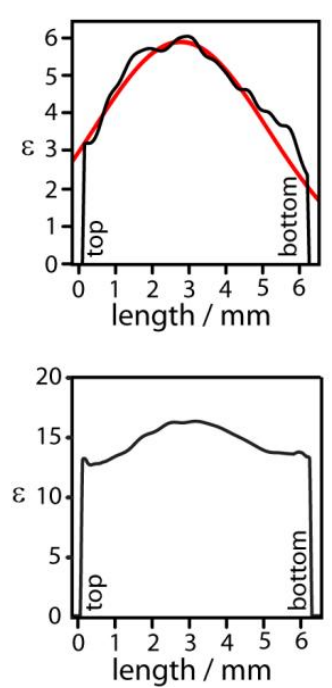

Figure 3. A comparison of DNP enhancement images (left) and cross-sections along the rotation axis (right) acquired on the same rotor under identical conditions prior to (a) and after (b) degassing. The degassing procedure not only improves the enhancement factors but also allows for a more uniform distribution of $\varepsilon$ values throughout the rotor. A red line in the plot in (a) also shows the microwave power distribution from the instrument's specifications.

A common procedure for improving the DNP polarization transfer is to degas the sample in the sample catcher by freezing and thawing the sample in a nitrogen atmosphere through repeated insertions and ejections.[23,27] Performing this procedure on the $\mathrm{Na}_{3} \mathrm{PO}_{4} \cdot 12 \mathrm{H}_{2} \mathrm{O}$ rotor indeed improved the DNP process such that the maximum DNP enhancement factor, measured at the center of the rotor, is now 16 instead of 6 . Interestingly, the degassing procedure also served to improve the uniformity of the DNP enhancement factors in the rotor such that the enhancement factors at the edges of the rotor are only $25 \%$ lower than in the center. 
(a) $120 \mathrm{~mA}$
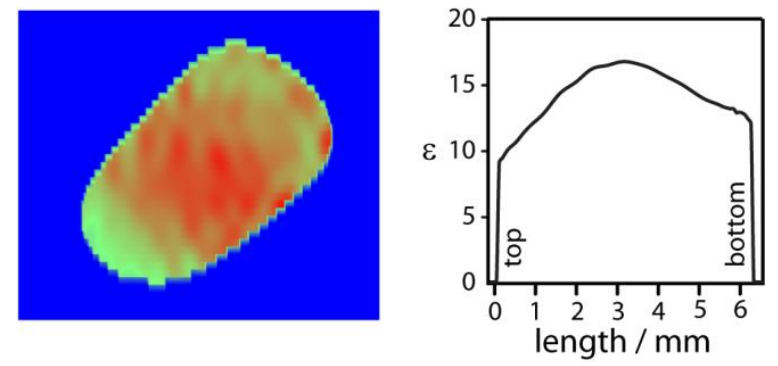

(b) $140 \mathrm{~mA}$
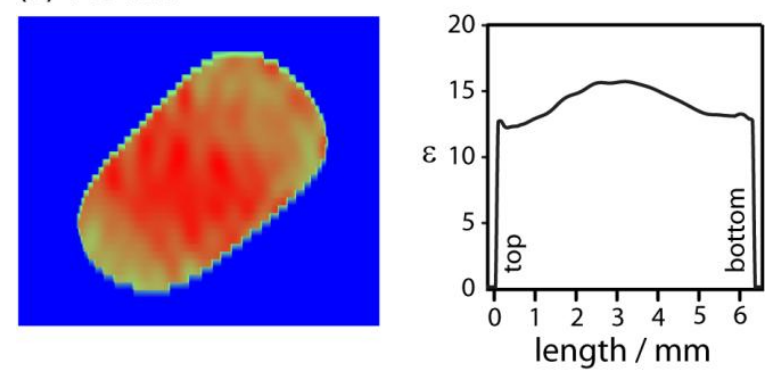

(c) $160 \mathrm{~mA}$
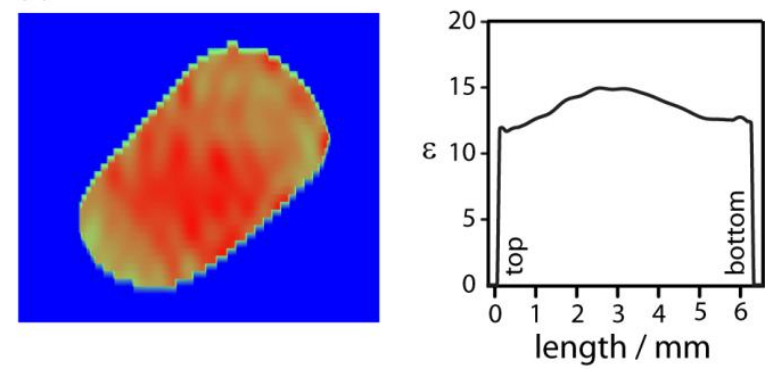

Figure 4. A comparison of DNP enhancement images (left) and cross-sections along the rotation axis (right) acquired on the same rotor using varying microwave power. A moving average was used to smooth the 1D images. The collector current value used in each experiment is indicated in the figure. An increase of the microwave power allows for the saturation of $\varepsilon$ values in a greater portion of the rotor.

Using this degassed sample, we also investigated the impact of varying the microwave power on the distribution of enhancement factors in the sample (Figure 4). The spatiallyaveraged DNP enhancement factors remained fairly constant at $\varepsilon=14$ as the gyrotron's collector current is increased from $120 \mathrm{~mA}$ to $140 \mathrm{~mA}$ and $160 \mathrm{~mA}$, which, according to a calorimeter 
located along the waveguide, corresponds to doubling the microwave power. Surprisingly, however, the lowest microwave power that was used led to the highest sensitivity in the center of the rotor, testifying to the very high microwave power in the part of the sample that is directly irradiated by the microwave beam. Increasing the microwave power didn't lead to larger enhancements in this part of the rotor whose $\varepsilon$ has already saturated. As the microwave power is increased, however, the enhancement factor at the top of the rotor increased from 9 to 13 , allowing for a greater fraction of the rotor to become polarized. The fact that the enhancement at center of the rotor is saturated under these conditions may explain the difference in enhancement distribution that is observed when comparing the images acquired prior to and after degassing the sample (Figure 3).

It has been recently demonstrated by Kubicki et al. that the efficiency of cross-effect DNP can be increased considerably by incorporating solid dielectric particles having sizes $>100$ $\mu \mathrm{m}$ into the frozen solutions.[27] For example, by adding powdered $\mathrm{KBr}$ or sapphire to a solution of TEKPol in TCE, the values of $\varepsilon$ increased from 228 to 449 and 515, respectively. Based on the simulations of microwave propagation, the effect was attributed to an increase of the average microwave field in the solution in the presence solid particles with a higher dielectric constant.[27] We have thus prepared a second rotor containing $\mathrm{Na}_{3} \mathrm{PO}_{4} \cdot 12 \mathrm{H}_{2} \mathrm{O}$ with $50 \%$ w/w of coarsely ground $\mathrm{KBr}$ crystals in order to experimentally determine the changes in the microwave power within the rotor that are induced by the dielectric particles. $\mathrm{KBr}$ particle sizes were on the order of $300( \pm 200) \mu \mathrm{m}$, as determined from an optical microscopy measurement.

The images in Figure 5 clearly show that the inclusion of $\mathrm{KBr}$ indeed strongly enhances the cross-effect. The spatially-averaged enhancement value of 36 and its peak value of 43 at the rotor's center are nearly 3 times larger than those measured in a rotor containing $100 \%$ 
$\mathrm{Na}_{3} \mathrm{PO}_{4} \cdot 12 \mathrm{H}_{2} \mathrm{O}$, which outweighs the lower sample content when $\mathrm{KBr}$ is added. Interestingly, the distribution of enhancement factors maintains the asymmetric profile, with a slightly higher amplification of the enhancement factors towards the bottom of the rotor, where these are 2.6 times larger, than at the top, where they are only 2.0 times larger. This fact is most clearly displayed in Figure $5 \mathrm{c}$ where the ratio of the enhancement factors, measured with and without $\mathrm{KBr}$, is plotted as a function of the position along the rotor's length. Most likely a non-uniform distribution of the two powders within the rotor, due the granular convection and the higher density of $\mathrm{KBr}$, is responsible for this effect. In agreement with the previously-proposed hypothesis,[27] it would then seem that $\mathrm{KBr}$ enhances the microwave power locally, by a repeated diffraction and refraction of microwaves, and does not help in obtaining a more uniform hyperpolarization. It may then be likely that at the center of the rotor in Kubicki's experiments enhancement factors exceeding 600 were obtained. 
(a) no $\mathrm{KBr}$ particles

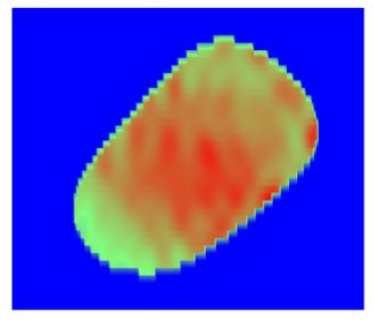

(b) $50 \% \mathrm{KBr}$ particles

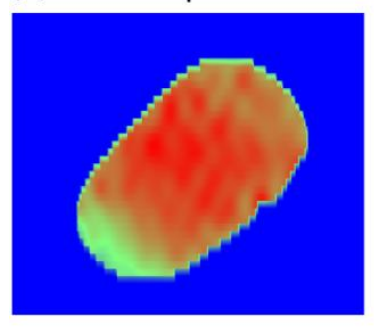

(c)

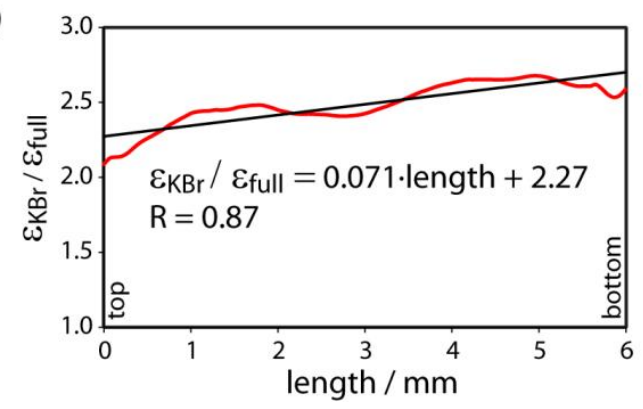

Figure 5. A comparison of DNP enhancement images (left) and cross-sections along the rotation axis (right) acquired on a rotor fully packed with $\mathrm{Na}_{3} \mathrm{PO}_{4} \cdot 12 \mathrm{H}_{2} \mathrm{O}$ (a) with one containing $50 \% \mathrm{w} / \mathrm{w}$ of coarsely ground $\mathrm{KBr}(\mathrm{b})$. A moving average was used to smooth the 1D images. The inclusion of dielectric particles increases the $\varepsilon$ values to a greater extent towards the bottom of the rotor. The ratio by which the $\varepsilon$ values are amplified is plotted in (c) as a function of the sample position along the rotation axis. A linear regression is used to highlight the trend.

\section{Conclusions}

We have demonstrated that the DNP enhancement factors within a full rotor spun at the magic angle can be mapped with the use of the STRAFI-MAS MRI experiment. These measurements provided interesting insights into the spatial distribution of the enhancement 
factors across the samples under various experimental conditions, which can provide guidance for expanding the limits of DNP, especially sample formulations and probe design.

We observed that the enhancement factors are largest in the center of the rotor, where the microwave beam is most intense. Surprisingly, the enhancement factors along the walls of the rotor are smaller than along its axis. We have also demonstrated that the use of higher microwave power and degassing of the sample allow for the saturation of the DNP enhancement factors in a greater fraction of the sample. Lastly, we have verified experimentally that the inclusion of large dielectric particles, such as $\mathrm{KBr}$ crystals, indeed leads to a local amplification of microwaves, but does not help in polarizing a greater fraction of the sample. Importantly, the size disparity between the sample and $\mathrm{KBr}$ leads to a gradient of enhancement amplification along the rotor. It is likely that the inclusion of an cylindrical Teflon lens to focalize the microwave beam along the length of the rotor, as has been previously proposed,[15] would lead to a more uniform enhancement of the sample and an improved sensitivity. Similarly, the use of smaller rotors should lead to a more uniform enhancement, as has been shown in recent simulations.[16] Improvements in the uniformity of the microwave power would also be needed in order implement pulsed DNP.[16] In light of these results, it would also seem that the reporting of DNP enhancement factors is highly subjective and, when doing so, it should be clearly stated whether a full rotor (maximising sensitivity) or a sparsely packed rotor (maximizing enhancement) is used.

\section{Acknowledgements}

We would like to thank Dr. Alan Wong for his useful discussions regarding the implementation of the STRAFI-MAS experiment. This research is supported by the U.S. Department of Energy 
(DOE), Office of Science, Basic Energy Sciences, Division of Chemical Sciences, Geosciences, and Biosciences. Support for F.P. is through a Spedding Fellowship funded by the LDRD program. Ames Laboratory is operated for the DOE by Iowa State University under Contract No. DE-AC02-07CH11358.

\section{References}

[1] R. G. Griffin, T. F. Prisner, High field dynamic nuclear polarization-the renaissance, Phys. Chem. Chem. Phys, 12 (2010) 5737-5740.

[2] Q. Z. Ni, E. Daviso, T. V. Can, E. Markhasin, S. K. Jawla, T. M. Swager, R. J. Temkin, J. Herzfeld, R. G. Griffin, High frequency dynamic nuclear polarization, Acc. Chem. Res. 46 (2013) 1933-1941.

[3] D. A. Hall, D. C. Maus, G. J. Gerfen, S. J. Inati, L. R. Becerra, F. W. Dahlquist, R. G. Griffin, Polarization-enhanced NMR spectroscopy of biomolecules in frozen solution, Science, 276 (1997) 930-932.

[4] R. G. Griffin, Clear signals from surfaces, Nature, 468 (2010) 381-382.

[5] A. J. Rossini, A. Zagdoun, M. Lelli, A. Lesage, C. Copéret, L. Emsley, Dynamic nuclear polarization surface enhanced NMR spectroscopy, Acc. Chem. Res. 46 (2013) 1942-1951.

[6] T. Kobayashi, F. A. Perras, I. I. Slowing, A. D. Sadow, M. Pruski, Dynamic nuclear polarization solid-state NMR in heterogeneous catalysis research, ACS Catal. 5 (2015) 7055-7062.

[7] J. H. Ardenkjaer-Larsen, B. Fridlund, A. Gram, G. Hansson, L. Hansson, M. H. Lerche, R. Servin, M. Thaning, K. Golman, Increase in signal-to-noise ratio of $>10,000$ times in liquidstate NMR, Proc. Nat. Acad. Sci. USA, 100 (2003) 10158-10163. 
[8] F. Blanc, L. Sperrin, D. Lee, R. Dervisoğlu, Y. Yamazaki, S. M. Haile, G. De Paëpe, C. P. Grey, Dynamic nuclear polarization NMR of low- $\gamma$ nuclei: structural insights into hydrated yttrium-doped $\mathrm{BaZrO}_{3}$, J. Phys. Chem. Lett. 5 (2014) 2431-2436.

[9] F. A. Perras, T. Kobayashi, M. Pruski, Natural abundance ${ }^{17}$ O DNP two-dimensional and surface-enhanced NMR spectroscopy, J. Am. Chem. Soc. 137 (2015) 8336-8339.

[10] M. Sharma, G. Janssen, J. Leggett, A. P. M. Kentgens, P. J. M. van Bentum, Rapid-melt dynamic nuclear polarization, J. Magn. Reson. 258 (2015) 40-48.

[11] R. P. Sangodkar, B. J. Smith, D. Gajan, A. J. Rossini, L. R. Roberts, G. P. Funkhouser, A. Lesage, L. Emsley, B. F. Chmelka, Influences of dilute organic adsorbates on the hydration of low-surface-area silicates, J. Am. Chem. Soc. 137 (2015) 8096-8112.

[12] L. R. Becerra, G. J. Gerfen, R. J. Temkin, D. J. Singel, R. G. Griffin, Dynamic nuclear polarization with a cyclotron resonance maser at 5 T, Phys. Rev. Lett. 71 (1993) 3561-3564.

[13] V. S. Bajaj, C. T. Farrar, M. K. Hornstein, I. Mastovsky, J. Vieregg, J. Bryant, B. Eléna, K. E. Kreischer, R. J. Temkin, R. G. Griffin, Dynamic nuclear polarization at $9 \mathrm{~T}$ using a novel 250 GHz gyrotron microwave source, J. Magn. Reson. 160 (2003) 85-90.

[14] M. Rosay, L. Tometich, S. Pawsey, R. Bader, R. Schauwecker, M. Blank, P. M. Borchard, S. R. Cauffman, K. L. Felch, R. T. Weber, R. J. Temkin, R. G. Griffin, W. E. Maas, Solidstate dynamic nuclear polarization at $263 \mathrm{GHz}$ : spectrometer design and experimental results, Phys. Chem. Chem. Phys. 12 (2010) 5850-5860.

[15] E. A. Nanni, A. B. Barnes, Y. Matsuki, P. P. Woskov, B. Corzilius, R. G. Griffin, R. J. Temkin, Microwave field distribution in a magic angle spinning dynamic nuclear polarization NMR probe, J. Magn. Reson. 210 (2011) 16-23. 
[16] D. E. M. Hoff, B. J. Albert, E. P. Saliba, F. J. Scott, E. J. Choi, M. Mardini, A. B. Barnes, Frequency swept microwaves for hyperfine decoupling and time domain dynamic nuclear polarization, Solid State Nucl. Magn. Reson. 72 (2015) 79-89.

[17] T. Maly, J. R. Sirigiri, Simplified THz Instrumentation for High-Field DNP-NMR Spectroscopy, Appl. Magn. Reson. 43 (2012) 181-194.

[18] K.-N. Hu, G. T. Debelouchina, A. A. Smith, R. G. Griffin, Quantum mechanical theory of dynamic nuclear polarization in solid dielectrics, J. Chem. Phys. 134 (2011) 125105.

[19] J. H. Baltisberger, S. Hediger, L. Emsley, Multi-dimensional magnetic resonance imaging in a stray magnetic field, J. Magn. Reson. 172 (2005) 79-84.

[20] A. Wong, D. Sakellariou, Constrast STRAFI-MAS imaging, J. Magn. Reson. 206 (2010) 264-268.

[21] A. Wong, D. Sakellariou, J. Magn. Reson. Imaging 32 (2010) 418-423.

[22] A. Wong, D. Sakellariou, Sensitivity-Enhanced Natural-Abundance Silicon-29 Magnetic Resonance Imaging, ChemPhysChem, 12 (2011) 3529-3532.

[23] A. Zagdoun, G. Casano, O. Ouari, M. Schwarzwälder, A. J. Rossini, F. Aussenac, M. Yulikov, G. Jeschke, C. Copéret, A. Lesage, P. Tordo, L. Emsley, Large molecular weight nitroxide biradicals providing efficient dynamic nuclear polarization at temperatures up to 200 K, J. Am. Chem. Soc. 135 (2013) 12790-12797.

[24] A. Wong, P. M. Aguiar, D. Sakellariou, Slow magic-angle coil spinning: A high-sensitivity and high-resolution NMR strategy for microscopic biological specimens, Magn. Reson. Med. 63 (2010) 269-274. 
[25] D. J. States, R. A. Haberkorn, D. J. Ruben, A two-dimensional nuclear Overhauser experiment with pure absorption phase in four quadrants, J. Magn. Reson. 48 (1982) 286292.

[26] D. Massiot, F. Fayon, M. Capron, I. King, S. Le Calvé, B. Alonso, J.-O. Durand, B. Bujoli, Z. Gan, G. Hoatson, Magn. Reson. Chem. 40 (2002) 70-76.

[27] D. J. Kubicki, A. J. Rossini, A. Purea, A. Zagdoun, O. Ouari, P. Tordo, F. Engelke, A. Lesage, L. Emsley, Amplifying dynamic nuclear polarization of frozen solutions by incorporating dielectric particles, J. Am. Chem. Soc. 136 (2014) 15711-15718. 